\title{
Correlation between brachial artery flow-mediated dilation and endothelial microparticle levels for identifying endothelial dysfunction in children with Kawasaki disease
}

\author{
Yue-Yue Ding', Yan Ren², Xing Feng ${ }^{3}$, Qiu-Qin Xu', Ling Sun', Jian-Min Zhang ${ }^{4}$, Ji-Juan Doư ${ }^{4}$, Hai-Tao Lvª and Wen-Hua Yan ${ }^{4}$
}

\begin{abstract}
BACKGROUND: We investigated vascular endothelial dysfunction by sonographic features of flow-mediated dilation (FMD) and circulating endothelial microparticles (EMPs) in Kawasaki disease (KD).
\end{abstract}

METHODS: Twenty-eight patients with KD were prospectively grouped according to stage of disease: acute, subacute, and convalescent. In addition, 28 healthy children and 28 febrile children were selected as controls. And cases in the convalescent phase were divided into two subgroups: coronary artery lesion (CAL) and no coronary lesion (NCAL). $\mathrm{CD}_{144}{ }^{+}$CD42b-, CD62E+, and CD105+ EMPs were measured by flow cytometry; FMD was obtained by sonography.

RESULTS: There were significant differences in FMD among the five groups. When compared with healthy controls, there were significantly greater numbers of CD144 $/ C D 42 b^{-}, C D 62 E^{+}$, and $\mathrm{CD}_{105^{+}}$EMPs and a higher proportion of CD62 $\mathrm{E}^{+} \mathrm{EMPs}$ in KD patients. The proportions and numbers of CD144 ${ }^{+} / \mathrm{CD}_{4} 2 \mathrm{~b}^{-}$, CD62E+, and CD105+ EMPs in KD patients were not statistically different than in febrile controls. There were no significant differences in FMD and EMPs between the CAL and NCAL subgroups. There were significantly negative correlations between the values of FMD and EMPs in the three phases of KD.

CONCLUSION: The increased levels of EMPs have significant correlation with decreased values of FMD, both of which may reflect endothelial dysfunction in child KD.

K awasaki disease (KD), also known as mucocutaneous lymph node syndrome, is an acute febrile disease that mostly strikes children aged 3 mo to $5 \mathrm{y}$. The main pathological changes are vasculitis of systemic small- and medium-sized arteries, especially the coronary arteries. In recent years, KD has replaced acute rheumatic fever as the most common cause of acquired heart disease in children of developed nations (1). As a developing country, China also observes a yearly increase in the incidence of $\mathrm{KD}(2)$. Acute self-limiting necrotizing arteritis, subacute/chronic vasculitis (SA/C), and luminal myofibroblastic proliferation are three characteristic vasculopathic processes in coronary and noncoronary arteries identified by light and transmission electron microscopy (3). Necrotizing arteritis, a synchronous neutrophilic process of the endothelium, beginning and ending within the first $2 \mathrm{wk}$ of fever onset, progressively destroys the wall into the adventitia, causing saccular aneurysms, which can then thrombose or rupture. SA/C vasculitis, an asynchronous process that commences in the first $2 \mathrm{wk}$, starts in the adventitia/perivascular tissue and variably inflames/damages the wall during its progression to the lumen. Besides causing fusiform and saccular aneurysms that can thrombose, SA/C vasculitis likely causes the transition of medial and adventitial smooth muscle cells into classic myofibroblasts. Luminal myofibroblastic proliferation is a progressive asynchronous intraluminal stenosing process of smooth muscle cell-derived myofibroblasts plus their matrix products and SA/C inflammation (SA/C-luminal myofibroblastic proliferation). Furthermore, SA/C-luminal myofibroblastic proliferation involves medium-sized arteries remote from active SA/C inflammation, possibly due to circulating factors, and also mildly involves subclinical veins, pulmonary arteries, and the aorta. In this context, we speculated that vasculitis in $\mathrm{KD}$ occurs in other vessels besides coronary endothelium. Endothelial dysfunction was found to be a long-term persistent condition in $\mathrm{KD}$ whether or not there is coronary arterial dilation (4-6). The evaluation of vascular endothelial function has important implications in early diagnosis, treatment, and prognosis in patients with $\mathrm{KD}$.

Recently, surrogate markers for coronary endothelial dysfunction were identified, which were associated with coronary aneurysm, atherosclerosis, and vascular function in KD. Flow-mediated dilation (FMD) reflecting vascular endothelial dysfunction has been reported in KD patients (7). Endothelial microparticles (EMPs) have been confirmed as biomarkers of endothelial dysfunction in KD patients $(8,9)$. But the relationship between FMD and EMP in signaling endothelial dysfunction has not been investigated. This study aims to assess vascular endothelial function in child $\mathrm{KD}$ patients using two indicators-imaging (FMD in the brachial artery by sonography) and serology (EMPs in blood). A correlation between

\footnotetext{
The first two authors contributed equally to this work.

'Department of Echocardiology, Children's Hospital Affiliated to Soochow University, Suzhou, Jiangsu Province, China; ${ }^{2}$ Department of Imaging, Children's Hospital Affiliated to Soochow University, Suzhou, Jiangsu Province, China; ${ }^{3}$ Institute of Pediatrics, Children's Hospital Affiliated to Soochow University, Suzhou, Jiangsu Province, China; ${ }^{4}$ Department of Cardiology, Children's Hospital Affiliated to Soochow University, Suzhou, Jiangsu Province, China.Correspondence: Hai-Tao Lv (haitaosz@163.com)

Received 4 April 2013; accepted 24 August 2013; advance online publication 29 January 2014. doi:10.1038/pr.2013.240
} 

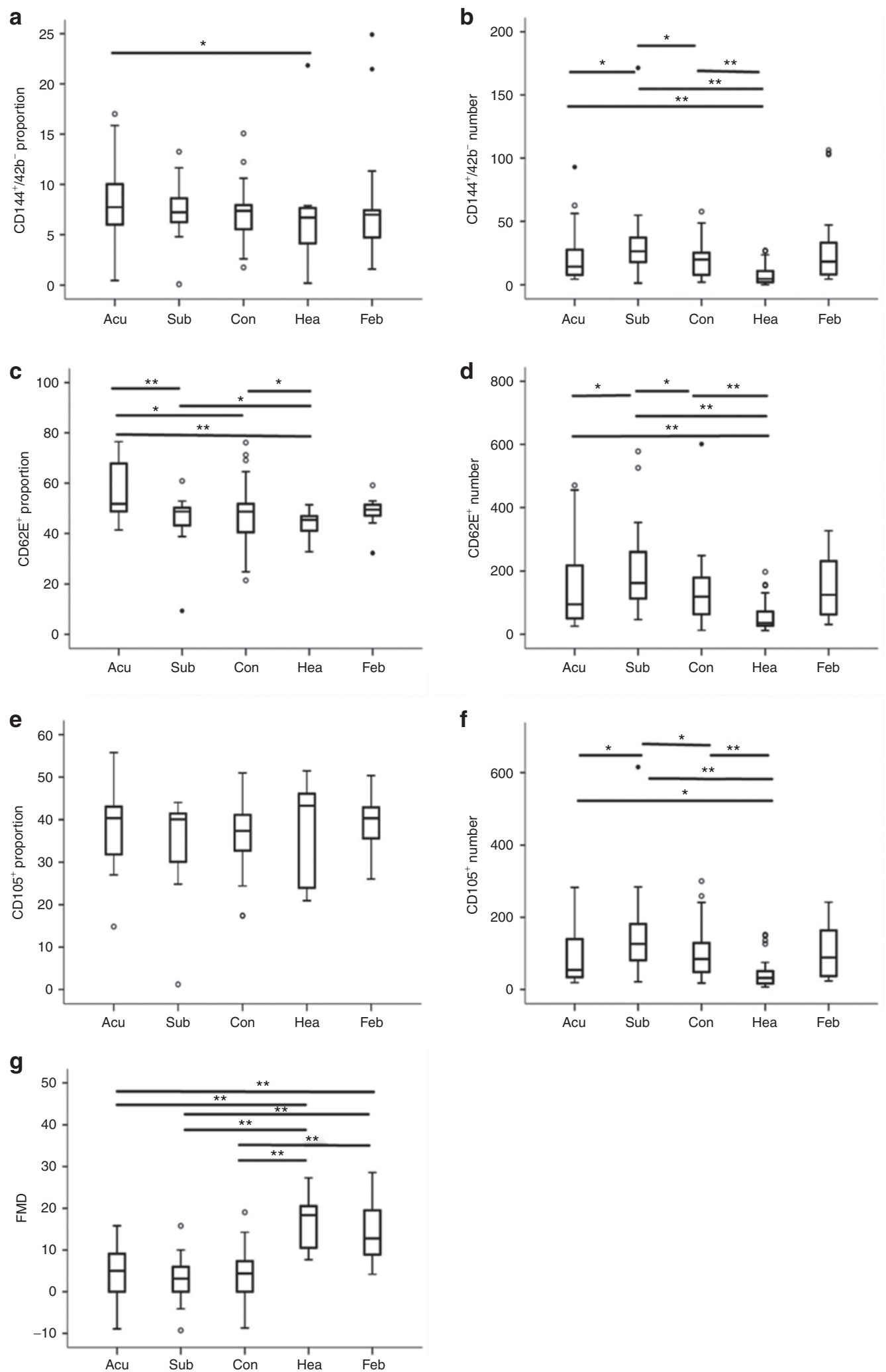

Figure 1. Levels of endothelial microparticles (EMPs) and flow-mediated dilation (FMD) in child Kawasaki disease patients. Acu, acute; Con, convalescent; Feb, febrile children (controls); Hea, healthy children (controls); Sub, subacute. Box plots show (a) the proportions of CD144 $/$ CD $_{2}$ b b $^{-}$EMPs, (b) the num-

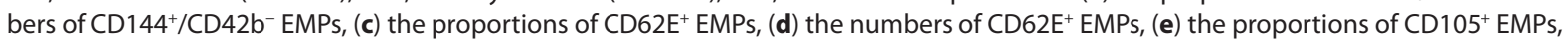

(f) the numbers of $\mathrm{CD} 105^{+}$EMPs, and (g) FMD. Results are expressed as events $\times 10^{6} / \mathrm{ml}$ of plasma and are given as mean \pm SD. The black lines represent comparisons between two groups and significance levels are indicated above each line $\left.{ }^{*} P<0.05 ;{ }^{* *} P<0.005\right)$. Open circles denote outlier cases, and filled circles denote extreme cases. 
brachial artery FMD and EMPs could establish a novel marker for identifying vascular endothelial dysfunction for KD.

\section{RESULTS}

\section{Clinical Features}

There was no significant difference in gender and age between $\mathrm{KD}$ cases, healthy controls, and febrile controls $(P>0.05)$. Out of $28 \mathrm{KD}$ patients, one did not respond to intravenous immunoglobulin (IVIG) and was subsequently given an intravenous pulse of methylprednisolone. Three KD patients presented with left ventricular dysfunction at admission, and nine with coronary aneurysm were given oral low-dose aspirin for 3-25 mo; the remaining patients were given standard treatment.

\section{Analysis of Levels of EMPs}

When compared among the five groups, the proportion of $\mathrm{CD}^{2} \mathrm{E}^{+} \mathrm{EMP}$ was significantly different, with $\chi^{2}$ values of 31.813 ( $P$ value $<0.001$ ); the numbers of $\mathrm{CD} 144^{+} / \mathrm{CD}^{2} 2 \mathrm{~b}^{-}$, $\mathrm{CD}^{2} \mathrm{E}^{+}$, and $\mathrm{CD} 105^{+} \mathrm{EMPs}$ were significantly different, with $\chi^{2}$ values of $37.737,35.089$, and 29.009 , respectively ( $P$ value $<$ 0.001; Figure 1a-f). Meanwhile, the proportions of $\mathrm{CD} 144^{+} /$ $\mathrm{CD}_{2} \mathrm{~b}^{-}$and $\mathrm{CD} 105^{+}$EMPs were not significantly different, with $\chi^{2}$ values of 7.907 and 4.046 ( $P$ value $\left.>0.05\right)$.

In acute-phase $\mathrm{KD}$, the proportions of $\mathrm{CD} 144^{+} / \mathrm{CD}_{2} 2 \mathrm{~b}^{-}$and $\mathrm{CD}_{2} \mathrm{E}^{+}$and the numbers of $\mathrm{CD} 144^{+} / \mathrm{CD} 42 \mathrm{~b}^{-}, \mathrm{CD} 62 \mathrm{E}^{+}$, and $\mathrm{CD} 105^{+}$EMPs were significantly higher when compared with healthy controls. In subacute and convalescent phases, the proportion of CD62 $\mathrm{E}^{+}$EMPs and the numbers of CD $144^{+} / \mathrm{CD} 42 \mathrm{~b}^{-}$, $\mathrm{CD} 62 \mathrm{E}^{+}$, and $\mathrm{CD} 105^{+} \mathrm{EMPs}$ were significantly increased.

Patients in any phase of $\mathrm{KD}$ had proportions and numbers of $\mathrm{CD} 144^{+} / \mathrm{CD}_{2} 2 \mathrm{~b}^{-}, \mathrm{CD}_{2} \mathrm{E}^{+}$, and $\mathrm{CD} 105^{+}$EMPs that were not statistically different from those of febrile controls $(P>0.05)$.

\section{EMP Levels in Three Phases of KD}

The numbers of $\mathrm{CD} 144^{+} / \mathrm{CD} 42 \mathrm{~b}^{-}, \mathrm{CD} 6 \mathrm{E}^{+}$, and $\mathrm{CD} 105^{+} \mathrm{EMPs}$ increased simultaneously with disease onset, rapidly reached a peak, and then decreased slowly, showing a monophasic course. In the subacute phase of KD, the numbers of $\mathrm{CD} 144^{+} /$ $\mathrm{CD}_{2} \mathrm{~b}^{-}, \mathrm{CD}_{105}{ }^{+}$, and $\mathrm{CD} 62 \mathrm{E}^{+}$EMPs significantly increased when compared with those in acute and convalescent phases $(P$ value $<0.05)$, and they were not significantly different between the acute and convalescent phases. The proportion of CD62 $\mathrm{E}^{+}$EMPs peaked initially in the acute phase and subsequently decreased in the following two phases. In acute-phase $\mathrm{KD}$, the proportion of $\mathrm{CD}_{2} \mathrm{E}^{+} \mathrm{EMPs}$ was significantly higher than that in subacute and convalescent phases $(P$ value $<0.05)$.

\section{FMD Among Cases and Controls}

There were significant differences in FMD values in the KD group among patients in the acute, subacute, and convalescent phases and between healthy and febrile controls $\left(\chi^{2}=69.713\right.$, $P<0.0001$; Figure 1g). The values of FMD were significantly lower in KD patients than in healthy and febrile controls but did not show significant differences in acute, subacute, and convalescent phases.

\section{Subjects in the Convalescent Phase of KD}

There were no significant differences in EMP or FMD values between 9 patients with coronary artery lesion (CAL) and 19 NCAL during the convalescent phase (Figure 2a).

\section{Correlation Between FMDs and EMPs}

FMD values were correlated with the stage of $\mathrm{KD}(r=0.577$; $P<0.005)$, coronary dilation $(r=-0.337 ; P<0.005)$, the number of CD $144^{+} / \mathrm{CD} 42 \mathrm{~b}^{-}$EMPs $(r=-0.504 ; P<0.005)$, the number of CD62 $\mathrm{E}^{+}$EMPs $(r=-0.493 ; P<0.005)$, and the number of $\mathrm{CD}_{105^{+}}$EMPs $(r=-0.503 ; P<0.005$; Figure $2 \mathbf{b}-\mathbf{d})$.

\section{DISCUSSION}

Celermajer et al. (10), in 1992, developed high-frequency ultrasound measurement of FMD in an artery as an indicator of endothelial vasodilatation function (11). When the cuff on the brachial artery is inflated, it occludes the artery and results in transient ischemia and hypoxia, as well as release of nitric oxide and other related vascular diastolic factors by the endothelium. In the normal condition of vascular endothelial function, reactive hyperemia of blood vessels occurs shortly following a block. Subsequently, nitric oxide and neurohormonal substances such as acetylcholine, serotonin, and catecholamines are released and these cause vasodilation. In patients with vascular endothelial dysfunction, the vascular response is impaired and diastolic capacity decreases. The low values of FMD in child KD patients represent a reduced ability for vasodilation, regardless of whether they are in the acute, subacute, or convalescent phase.

EMPs released from endothelial plasma membrane blebbing carry endothelial proteins such as vascular endothelial adhesion molecule (CD144), platelet endothelial cell adhesion molecule-1 (CD31), intercellular cell adhesion molecule-1 (CD54), endoglin (CD105), E-selectin (CD62E), S-endo antigen (CD146), and $\alpha v$ integrin (CD51). Identification of endothelial origin of circulating microparticles relies on the use of specific markers in flow cytometry analysis. CD62E is expressed by activated endothelial cells and CD105 in activated monocytes/macrophages and bone marrow cell subsets. CD144 is specifically expressed in the endothelial cells (12). EMPs externalize phosphatidylserine and bind annexin $\mathrm{V}$ in a calcium-dependent manner. The protein composition of EMP highly depends on the stimulus triggering their release. EMPs play important roles in inflammation, coagulation, and regulation of vascular function. Significantly elevated levels of plasma EMPs were reported in cardiovascular diseases with endothelial dysfunction, such as acute coronary syndrome (13), pulmonary hypertension (14), diabetes (15), vasculitis (16), and others $(17,18)$.

The characteristics of circulating EMPs, during different stages of KD in children, have been rarely investigated. Guiducci et al. (8) reported increased numbers of microparticles in $\mathrm{KD}$ patients, mainly from endothelial cells and T cells, following IVIG therapy. Tan et al. (9) also found elevated EMP levels in KD patients, as well as a positive correlation with tumor necrosis factor- $\alpha$ and a negative correlation with albumin. In 
a

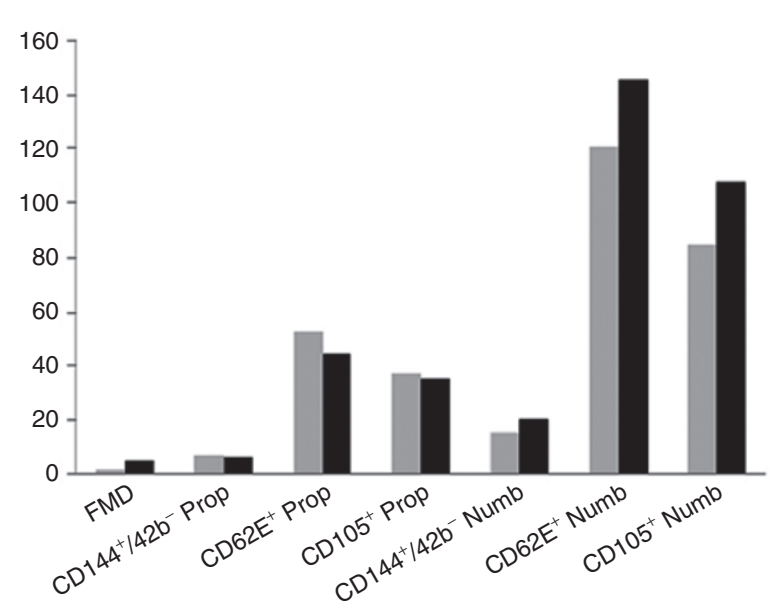

C

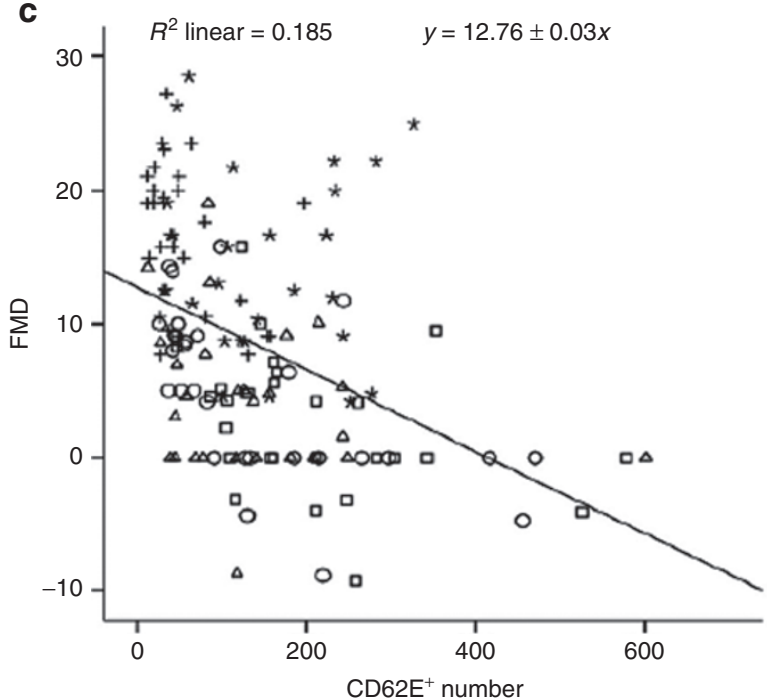

b

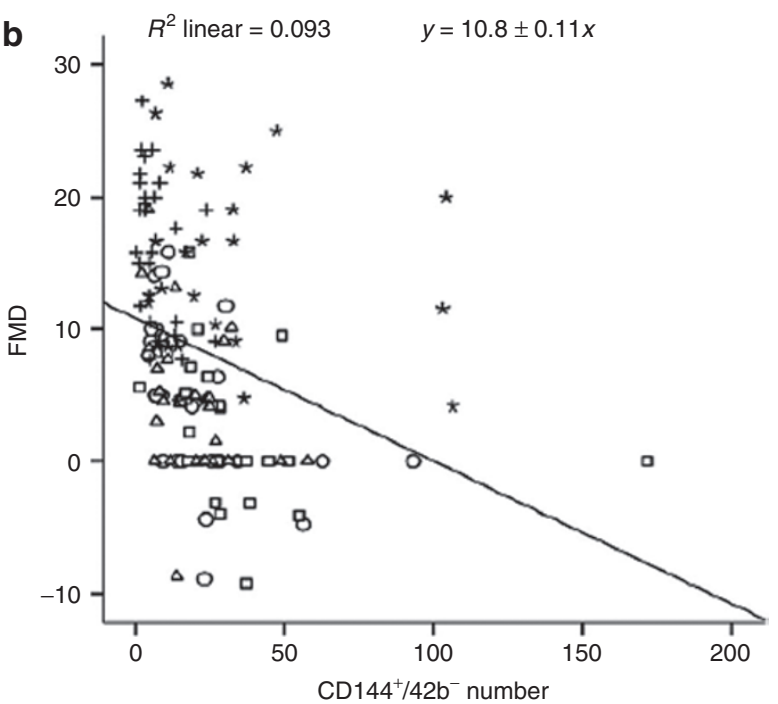

d

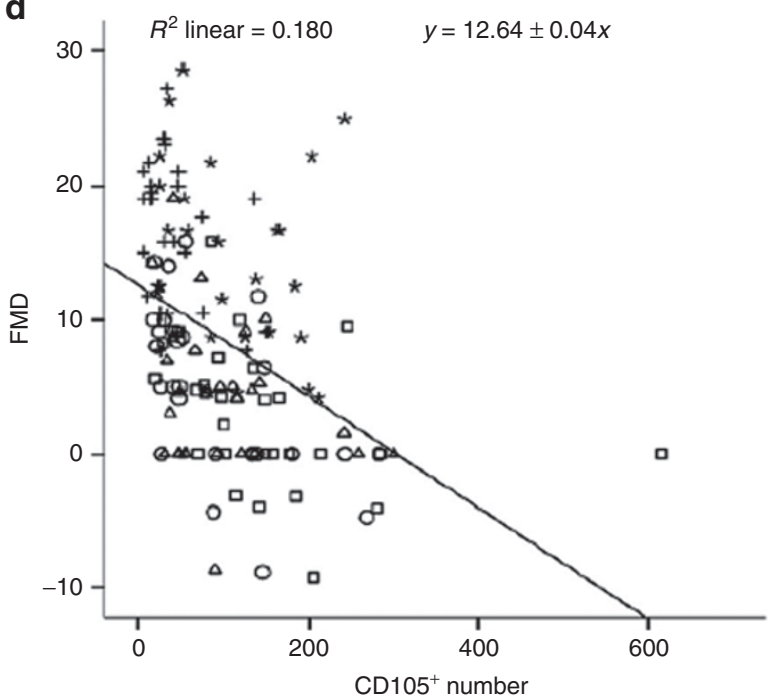

Figure 2. Comparisons of flow-mediated dilation (FMD) and endothelial microparticles (EMPs) between patients with and without coronary artery disease (CAL) in the convalescent phase of Kawasaki disease (KD) and correlations between FMD and EMP values in five groups. (a) There were no significant differences in FMD or EMP values between patients with and without CAL (columns of gray and black denote CAL and no CAL, respectively) in the

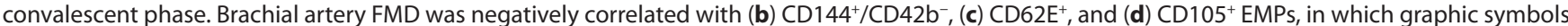
of open circle, open square, open triangle, $t,{ }^{*}$ denote the five groups of acute, subacute, convalescent, healthy, and febrile, respectively.

this study, we found much higher levels of $\mathrm{CD} 144^{+} / \mathrm{CD} 42 \mathrm{~b}^{-}$, $\mathrm{CD}_{2} \mathrm{E}^{+}$, and $\mathrm{CD}_{105^{+}} \mathrm{EMPs}$ in KD patients than in healthy children. The proportion of CD62E EMPs initially peaked and slowly declined, suggesting involvement of EMPs in the development of vasculitis in children with KD. The generation of EMPs is understood, but the metabolism or clearing pathway of these EMPs is unclear, especially in different phases of KD. We observed changes in EMPs during different phases of KD, but the time of follow-up was too short to explore the reason and mechanism for reduction in their numbers.

When compared with febrile controls, no remarkable elevation in proportions and numbers of EMPs were found, probably because of the release of inflammatory mediators involving vascular endothelium in respiratory infections. The generation of EMPs from human umbilical vein endothelial cells after activation through stimulation of tumor necrosis factor- $\alpha$ and other inflammatory cytokines has also been reported (12). Meanwhile, in KD patients, the histological characteristic of vasculitis mainly includes proliferative granulomatous inflammation, consisting of marked immune activation and subsequent release of inflammatory cytokines such as interleukin-1 and tumor necrosis factor- $\alpha$ (19). Therefore, the differentiation of EMPs between KD and other febrile diseases may be difficult.

We observed increased levels of EMPs, whether for activation or apoptosis, which may lend credence to the hypothesis that both activation and apoptosis contribute to the vascular injury associated with KD in young children. Particularly, the remarkable increase in $\mathrm{CD}_{2} \mathrm{E}^{+}$levels suggested that activated endothelial cells were predominant in patients with $\mathrm{KD}$. So we believe that the level of $\mathrm{CD}^{2} 2 \mathrm{E}^{+}$EMPs could be a helpful marker 
for KD. Clarke et al. (20) concluded that changes in the level of EMPs are mainly determined by the activity of disease but not by the treatment. We also found elevated levels of circulating EMPs in children with KD during the acute phase and relatively higher levels even in the subacute phase after treatment.

In the convalescent phase of $\mathrm{KD}$, there were no statistical differences in the levels of EMPs or FMD values between CAL and NCAL patients. For FMD values, our study differs in part at least from other studies reporting significant difference of FMD values between CAL and NCAL groups $(6,21)$; the reason could be our small sample size. Regarding EMP values, there were no similar study reports. We believe that the endothelial damage both with and without CAL persists into the chronic stage of $\mathrm{KD}$, and the occurrence of CAL is influenced by the genetic background (22). Further studies with a larger sample size are required to confirm the significance of this observation.

So far, the correlation between EMPs and FMD in KD patients has not been reported, although their relation has been studied in end-stage renal disease (23), diabetes (15), ischemic left ventricular function insufficiency $(24)$, and obesity $(16,25)$. During end-stage renal disease, circulating microparticles of endothelial origin are tightly associated with endothelial dysfunction and arterial dysfunction (23). In diabetics, FMD is negatively correlated with $\mathrm{CD} 1^{+} / \mathrm{CD} 42^{-}$EMPs and $\mathrm{CD} 51^{+}$EMPs (15). The study of Bulut et al. (24) demonstrated that EMPs are inversely correlated with endothelial dysfunction, as assessed by FMD, in patients with ischemic left ventricular function insufficiency. FMD is a mature imaging parameter, which highly correlates with coronary endothelial function. This study showed that FMD correlated with levels of circulating $\mathrm{CD} 144^{+} / \mathrm{CD}_{2} 2 \mathrm{~b}^{-}$, $\mathrm{CD}_{22} \mathrm{E}^{+}$, and $\mathrm{CD} 105^{+}$microparticles and that these EMPs could independently reflect changes of the vascular endothelial function in children with KD. The combination of FMD and EMPs as a diagnostic tool in routine clinical practice is not yet warranted, but in the absence of reliable tools for diagnosing $\mathrm{KD}$, this investigation could prove useful in the future.

\section{Conclusion}

FMD of the brachial artery is a noninvasive method to assess the status of vascular endothelial function. EMP levels in the plasma of $\mathrm{KD}$ patients were significantly higher in the acute and subacute phases and were lower during the convalescent phase. EMP levels remained higher in KD patients than in healthy children, suggesting that the endothelial damage persisted during the convalescent phase of $\mathrm{KD}$. The correlation between the levels of EMPs and brachial artery FMD suggests that their combination could serve as an indicator of endothelial dysfunction in patients with KD.

\section{METHODS}

\section{Patients and Controls}

We performed a prospective, case-control study that included 28 KD patients and 56 controls including 28 healthy and 28 febrile patients. KD patients were diagnosed by the criteria established by the Japanese Kawasaki Disease Research Committee (26). All $28 \mathrm{KD}$ patients (16 males and 12 females) were recruited from the
Department of Cardiology, Soochow University Affiliated Children's Hospital (Suzhou, Jiangsu Province, China) between March 2009 and December 2011. Their median age was 23 mo (range: 4-90 mo). All of these patients were individually evaluated in the acute, subacute, and convalescent phases of KD. Twenty-eight healthy children, 16 males and 12 females, with a median age of 22 mo (range: 3-87 mo), were selected as healthy controls. Twenty-eight febrile children, 14 males and 14 females, with a median age of 22 mo (range: 5-90 mo) and proven respiratory infection, were selected as febrile controls. The Soochow University Affiliated Children's Hospital ethics committee approved the study. An informed written consent was obtained from the parents of each patient.

\section{Determination of CALs}

CALs mainly consist of occlusion, segmental stenosis, localized stenosis, dilation, and aneurysm of the coronary artery during angiography (27). In this study, only dilation and aneurysms were detected by echocardiography at admission, at the second week of illness, and then at 1, 3, 6, and 12 mo. The maximum internal diameters of both the right and left coronary arteries were measured. Those that were greater than $2.5 \mathrm{~mm}$ in patients aged $<3 \mathrm{y}$, greater than $3.0 \mathrm{~mm}$ in patients aged 3-9 $\mathrm{y}$, and greater than $3.5 \mathrm{~mm}$ in patients aged $>9 \mathrm{y}$ were considered to have CAL (28).

\section{Treatment Protocol of KD}

Standard treatment given for KD was as follows (29): patients were treated with IVIG $(2 \mathrm{~g} / \mathrm{kg})$ in a single infusion, together with highdose aspirin administered at $30-50 \mathrm{mg} / \mathrm{kg} /$ day if they were presented within the first 5-10 d of illness. Repeat treatment with IVIG was considered if patients had persistent fever without other explanations, $48 \mathrm{~h}$ after initial therapy. High-dose aspirin was continued until $\geq 48$ $72 \mathrm{~h}$ after fever cessation. Then, low-dose aspirin (3-5 mg/kg/day) was initiated and maintained until the patient showed no evidence of coronary changes for $6-8 \mathrm{wk}$ after the onset of illness. For children who developed coronary abnormalities, aspirin was continued indefinitely. Steroid treatment was restricted to children in whom $\geq 2$ infusions of IVIG had been ineffective in alleviating fever and acute inflammation. The steroid regimen consisted of intravenous pulse of methylprednisolone at $30 \mathrm{mg} / \mathrm{kg}$ for $2-3 \mathrm{~h}$, administered once daily for $1-3 \mathrm{~d}$.

\section{Assessment of Brachial Arterial FMD}

The method developed by Celermajer in 1992 was used to measure flow-mediated vasodilation $(7,10)$. A single sonographer examined all subjects in a quiet condition; chloral hydrate $(10 \%$ solution at $0.5 \mathrm{ml} /$ kg body weight) was used to sedate uncooperative subjects. A ultrasound machine (GE vivid 7) with a 9L transducer $(6-10 \mathrm{MHz})$ was used to detect the brachial artery above the antecubital fossa of the right arm. Longitudinal two-dimensional images were acquired to measure the distance between the anterior and the posterior intima of the vascular wall at the end of diastole $\left(D_{1}\right)$ as a baseline internal diameter. Then the brachial artery was occluded by inflating the blood pressure cuff to $50 \mathrm{mmHg}$ above the subject's resting systolic blood pressure. The cuff remained inflated for $5 \mathrm{~min}$ and was then quickly deflated. Sixty seconds later, the diastolic diameter $\left(D_{2}\right)$ was again obtained. The same sonographer measured all of the indicators, and the value of FMD was calculated using the following formula:

$\mathrm{FMD}=\left[\left(D_{2}-\mathrm{D}_{1}\right) / \mathrm{D}_{1}\right] \times 100 \%$

\section{Measurement of EMPs}

Blood samples were initially centrifuged in ethylene diamine tetraacetic acid tubes ( $10 \mathrm{~min} ; 3,000 \mathrm{~g}$ ) to obtain platelet-rich plasma and then ultracentrifuged $(10 \mathrm{~min} ; 12,000 \mathrm{~g})$ to get platelet-poor plasma. Each tube of platelet-poor plasma $(50 \mu \mathrm{l})$, preloaded with fluorescent TruCount bead lyophilized pellets (Becton-Dickinson Biosciences, San Jose, CA), was added to $10 \mu \mathrm{l}$ of CD62E-PE (phycoerythrin), CD144-FITC (fluorescein isothiocyanate), CD42b-APC (allophycocyanin) individually, and to $5 \mu \mathrm{l}$ of CD105-PerCP-Cy5.5 (PeridininChlorophyll protein with a cyanine dye); antibodies were obtained from Becton-Dickinson Biosciences. The mixtures were subsequently incubated at room temperature, away from light for $20 \mathrm{~min}$, followed by the addition of phosphate-buffered saline solution $(420 \mu \mathrm{l})$ for analysis 
by flow cytometry (FACs Calibur, Becton-Dickinson Biosciences). Meanwhile, isotype controls, labeled with PE, FITC, APC, and PerCP-Cy5.5 antibodies, were used for eliminating background staining produced by nonspecific binding of the antibody. Instrument settings were adjusted by analyzing $0.8 \mu \mathrm{m}$ latex beads (Sigma-Aldrich, St Louis, MO). Gates were positioned to include particles less than $0.8 \mu \mathrm{m}$ in size. EMPs were defined as $\mathrm{CD} 144^{+} / \mathrm{CD}_{42} \mathrm{~b}^{-}, \mathrm{CD} 105^{+}$, and $\mathrm{CD} 62 \mathrm{E}^{+}$ microparticles. Counting stopped when 10,000 particles were collected, and the proportions of EMPs were calculated automatically. The numbers of EMPs were calculated according to the following formula:

Number of EMPs $/ \mathrm{ml}=$ number of events in EMP region $(\mathrm{R} 5) \times$ total number of beads per tube/number of beads collected (R3) $\times$ test volume $(50 \mu \mathrm{l})$

\section{Statistical Analysis}

Data were analyzed using SPSS 17.0 (SPSS, Chicago, IL) statistical software. Results were presented as mean \pm SD. The values of EMPs and FMD were analyzed using the Kruskal-Wallis $H$-test and MannWhitney $U$-test. The correlation between the values of FMD and levels of EMPs was analyzed by Spearman's rank correlation. $P<0.05$ was considered statistically significant.

\section{STATEMENT OF FINANCIAL SUPPORT}

This work was financially supported by the Jiangsu Province Science Foundation (BE2013632), Jiangsu Province Health Department (H201127), Suzhou Science and Technology Bureau (SYS201144, SYS201044, KJXW2011019), Jiangsu Health International Exchange Program (2011), and the Suzhou Child Development and Brain Injury Research Key Laboratory Foundation (SZS201108).

Disclosure: The authors declare that they have no conflict of interest.

\section{ADDITIONAL AUTHOR INFORMATION}

Y.-Y.D. carried out the studies, participated in the sonographic detection of flow-mediated dilation, and drafted the manuscript. Y.R., as a coauthor, conceived the study, participated in its design and coordination, and helped to draft the manuscript. Q.-Q.X. carried out the sonographic detecting of flowmediated dilation. X.F., L.S., J.-M.Z., and W.-H.Y. participated in the collection of clinical cases. J.-J.D. performed the statistical analysis. H.-T.L., as the corresponding author, participated in the design of the whole study and guided the experimental process. All authors read and approved the final manuscript.

\section{REFERENCES}

1. Gordon JB, Kahn AM, Burns JC. When children with Kawasaki disease grow up: myocardial and vascular complications in adulthood. J Am Coll Cardiol 2009;54:1911-20.

2. Ma XJ, Yu CY, Huang M, Chen SB, Huang MR, Huang GY; Shanghai Kawasaki Research Group. Epidemiologic features of Kawasaki disease in Shanghai from 2003 through 2007. Chin Med J 2010;123:2629-34.

3. Orenstein JM, Shulman ST, Fox LM, et al. Three linked vasculopathic processes characterize Kawasaki disease: a light and transmission electron microscopic study. PLoS ONE 2012;7:e38998.

4. Mitani Y. Coronary endothelial dysfunction after Kawasaki disease. J Am Coll Cardiol 2000;35:821-3.

5. Fukazawa R. Long-term prognosis of Kawasaki disease: increased cardiovascular risk? Curr Opin Pediatr 2010;22:587-92.

6. Ikemoto Y, Ogino H, Teraguchi M, Kobayashi Y. Evaluation of preclinical atherosclerosis by flow-mediated dilatation of the brachial artery and carotid artery analysis in patients with a history of Kawasaki disease. Pediatr Cardiol 2005;26:782-6.

7. Urbina EM, Williams RV, Alpert BS, et al.; American Heart Association Atherosclerosis, Hypertension, and Obesity in Youth Committee of the Council on Cardiovascular Disease in the Young. Noninvasive assessment of subclinical atherosclerosis in children and adolescents: recommendations for standard assessment for clinical research: a scientific statement from the American Heart Association. Hypertension 2009;54:919-50.

8. Guiducci S, Ricci L, Romano E, et al. Microparticles and Kawasaki disease: a marker of vascular damage? Clin Exp Rheumatol 2011;29:Suppl 64:S121-5.
9. Tan Z, Yuan Y, Chen S, Chen Y, Chen TX. Elevated plasma endothelial microparticles together with TNF-alpha and IL-6 in Kawasaki Disease. Indian Pediatr 2013;50:501-3.

10. Celermajer DS, Sorensen KE, Gooch VM, et al. Non-invasive detection of endothelial dysfunction in children and adults at risk of atherosclerosis. Lancet 1992;340:1111-5.

11. Santos-García D, Rodríguez-Yáñez M, Arias-Rivas S, Blanco M. [Brachial arterial flow mediated dilation: utility in clinical and experimental practice]. Rev Neurol 2011;53:351-60.

12. Dignat-George F, Boulanger CM. The many faces of endothelial microparticles. Arterioscler Thromb Vasc Biol 2011;31:27-33.

13. Biasucci LM, Porto I, Di Vito L, et al. Differences in microparticle release in patients with acute coronary syndrome and stable angina. Circ J 2012;76:2174-82.

14. Amabile N, Heiss C, Real WM, et al. Circulating endothelial microparticle levels predict hemodynamic severity of pulmonary hypertension. Am J Respir Crit Care Med 2008;177:1268-75.

15. Feng B, Chen Y, Luo Y, Chen M, Li X, Ni Y. Circulating level of microparticles and their correlation with arterial elasticity and endothelium-dependent dilation in patients with type 2 diabetes mellitus. Atherosclerosis 2010;208:264-9.

16. Parker B, Al-Husain A, Pemberton P, et al. Suppression of inflammation reduces endothelial microparticles in active systemic lupus erythematosus. Ann Rheum Dis 2013; e-pub ahead of print 5 May 2013.

17. Bulut D, Tüns H, Mügge A. CD31+/Annexin V+ microparticles in healthy offsprings of patients with coronary artery disease. Eur J Clin Invest 2009;39:17-22.

18. Lackner P, Dietmann A, Beer R, et al. Cellular microparticles as a marker for cerebral vasospasm in spontaneous subarachnoid hemorrhage. Stroke 2010;41:2353-7.

19. Takahashi K, Oharaseki T, Yokouchi Y, Hiruta N, Naoe S. Kawasaki disease as a systemic vasculitis in childhood. Ann Vasc Dis 2010;3:173-81.

20. Clarke LA, Hong Y, Eleftheriou D, et al. Endothelial injury and repair in systemic vasculitis of the young. Arthritis Rheum 2010;62:1770-80.

21. Niboshi A, Hamaoka K, Sakata K, Yamaguchi N. Endothelial dysfunction in adult patients with a history of Kawasaki disease. Eur J Pediatr 2008;167:189-96.

22. Onouchi Y. Molecular genetics of Kawasaki disease. Pediatr Res 2009;65(5 Pt 2):46R-54R.

23. Amabile N, Guérin AP, Leroyer A, et al. Circulating endothelial microparticles are associated with vascular dysfunction in patients with end-stage renal failure. J Am Soc Nephrol 2005;16:3381-8.

24. Bulut D, Maier K, Bulut-Streich N, Börgel J, Hanefeld C, Mügge A. Circulating endothelial microparticles correlate inversely with endothelial function in patients with ischemic left ventricular dysfunction. J Card Fail 2008; $14: 336-40$.

25. Esposito K, Ciotola M, Schisano B, et al. Endothelial microparticles correlate with endothelial dysfunction in obese women. J Clin Endocrinol Metab 2006;91:3676-9.

26. Ayusawa M, Sonobe T, Uemura S, et al.; Kawasaki Disease Research Committee. Revision of diagnostic guidelines for Kawasaki disease (the 5th revised edition). Pediatr Int 2005;47:232-4.

27. Suzuki A, Kamiya T, Kuwahara N, et al. Coronary arterial lesions of Kawasaki disease: cardiac catheterization findings of 1100 cases. Pediatr Cardiol 1986;7:3-9..

28. Kobayashi T, Inoue Y, Takeuchi K, et al. Prediction of intravenous immunoglobulin unresponsiveness in patients with Kawasaki disease. Circulation 2006;113:2606-12.

29. Newburger JW, Takahashi M, Gerber MA, et al.; Committee on Rheumatic Fever, Endocarditis, and Kawasaki Disease, Council on Cardiovascular Disease in the Young, American Heart Association. Diagnosis, treatment, and long-term management of Kawasaki disease: a statement for health professionals from the Committee on Rheumatic Fever, Endocarditis, and Kawasaki Disease, Council on Cardiovascular Disease in the Young, American Heart Association. Pediatrics 2004;114:1708-33. 